Publ. RIMS, Kyoto Univ.

12 Suppl. (1977), 233-245.

\title{
Irregularity of Characteristic Elements and Hyperbolicity ${ }^{1)}$
}

\author{
by
}

Hikosaburo Komatsu*

\section{$\S 1$. Irregularity of Characteristic Elements.}

Let

$$
P(x, D)=\sum_{|\alpha| \leqq m} a_{a}(x) D^{\alpha}
$$

be a linear partial differential operator with holomorphic coefficients $a_{\alpha}(x)$ defined on an open set $V$ in $\mathbb{C}^{n}$. We denote the principal part by $p(x, D)$ :

$$
p(x, D)=\sum_{|\alpha|=m} a_{\alpha}(x) D^{\alpha}
$$

and assume that it is not degenerate or that $p(x, D) \neq 0$ for any fixed $x$ in $V$. Since we consider only local problems, we may assume without loss of generality that the coefficient of $D_{1}{ }^{m}$ is equal to 1 .

If an element $\left(x^{0}, \xi^{0} \infty\right) \in P^{*} V=\left(T^{*} V-V\right) / \mathbb{C}^{<}\left(\xi^{0} \infty\right.$ stands for the class of $\left.\xi^{0} \in T_{x^{0}}^{*} V\right)$ is on the non-singular part of the variety

$$
N(p)=\left\{(x, \xi \infty) \in P^{*} V ; p(x, \xi)=0\right\},
$$

it is called a non-singular characteristic element of $P(x, D)$. Then $p(x, \xi)$ is factorized in a neighborhood of $\left(x^{0}, \xi^{0}\right)$ as

$$
p(x, \xi)=\rho(x, \xi)\left(\xi_{1}-\lambda\left(x, \xi^{\prime}\right)\right)^{d}
$$

with holomorphic functions $\rho(x, \xi)$ and $\lambda\left(x, \xi^{\prime}\right)$ homogeneous in $\xi$ and such that $\rho\left(x^{0}, \xi^{0}\right) \neq 0$. The integer $d$ is the multiplicity of the characteristic element.

Let $\mathcal{O}$ be the ring of germs of holomorphic functions on a neighborhood

Received October 9, 1976.

* Department of Mathematics, Faculty of Science, University of Tokyo, Hongo, Tokyo, Japan.

1) Preliminary results were announced at the conference on Structure of Solutions of Partial Differential Equations at RIMS on October 24, 1975. 
of $x^{0}$ and let $\mathcal{O}[\xi]$ be the ring of polynomials with coefficients in $\mathcal{O}$. These rings are known to be unique factorization domains. Therefore we can find an irreducible homogeneous polynomial $K(x, \xi)$ in $\mathcal{O}[\xi]$ such that $\xi_{1}-\lambda\left(x, \xi^{\prime}\right)$ divides $K(x, \xi)$ in a neighborhood of $\left(x^{0}, \xi^{0}\right)$. The irreducibility of $K(x, \xi)$ implies that $\xi_{1}-\lambda\left(x, \xi^{\prime}\right)$ is a simple factor of $K(x, \xi)$ and that if $\xi_{1}-\lambda\left(x, \xi^{\prime}\right)$ divides a polynomial $A(x, \xi)$ in $\mathcal{O}[\xi]$ as a holomorphic function, then $K(x, \xi)$ divides $A(x, \xi)$ in $\mathcal{O}[\xi]$ (see [17]). In particular, there is a homogeneous polynomial $Q(x, \xi)$ in $\mathcal{O}[\xi]$ such that

$$
p(x, \xi)=Q(x, \xi) K(x, \xi)^{d} .
$$

We have $Q\left(x^{0}, \xi^{0}\right) \neq 0 . \quad P(x, D)-Q(x, D) K(x, D)^{d}$ is a differential operator of order at most $m-1$. If its homogeneous part $P^{m-1}(x, D)$ of order $m-1$ is equal to zero, we set $d_{m-1}=\infty$ and $Q_{m-1}(x, D)=0$. Otherwise, we can factorize $P^{m-1}(x, \xi)$ in $\mathcal{O}[\xi]$ as

$$
P^{m-1}(x, \xi)=Q_{m-1}(x, \xi) K(x, \xi)^{d_{m-1}},
$$

so that $Q_{m-1}(x, \xi)$ does not vanish identically on a neighborhood of ( $x^{0}$, $\left.\xi^{0}\right)$ in the zeros $N(K)$ of $K$ or of $\xi_{1}-\lambda\left(x, \xi^{\prime}\right)$. We continue the same procedure up to order zero and obtain the following decomposition of $P(x, D)$ due to De Paris [4]:

$$
\begin{aligned}
P(x, D)=Q & (x, D) K(x, D)^{d}+Q_{m-1}(x, D) K(x, D)^{d_{m-1}} \\
& +\cdots+Q_{0}(x, D) K(x, D)^{d_{0}}
\end{aligned}
$$

where $Q_{i}(x, D) K(x, D)^{d_{i}}$ is either zero or a differential operator of order $i$ with a homogeneous polynomial $Q_{i}(x, \xi)$ which does not vanish identically on a neighborhood of $\left(x^{0}, \xi^{0}\right)$ in $N(K)$.

Now the irregularity $\sigma$ of the characteristic element $\left(x^{0}, \xi^{0} \infty\right.$ ) (or the factor $K(x, D))$ is defined by

$$
\sigma=\max \left\{1, \frac{d-d_{i}}{m-i}(i=0,1, \cdots, m-1)\right\}
$$

We remark that $1 \leqq \sigma \leqq d$. The De Paris decomposition (6) depends on the coordinate system but the irregularity does not.

When $\sigma=1, P(x, D)$ is said to satisfy Levi's condition at $\left(x^{0}, \xi^{0} \infty\right)$. 


\section{$\S 2$. Estimates of Coefficients of Formal Solutions.}

A holomorphic function $\varphi(x)$ on a neighborhood of $x^{0}$ is called a characteristic function of $P(x, D)$ if it satisfies

$$
p(x, \operatorname{grad} \varphi(x))=0 .
$$

We assume that $\operatorname{grad} \varphi\left(x^{0}\right) \infty=\xi^{0} \infty$. Then $\varphi(x)$ is actually a solution of

$$
\frac{\partial \varphi(x)}{\partial x_{1}}-\lambda\left(x, \frac{\partial \varphi(x)}{\partial x^{\prime}}\right)=0 .
$$

Let $\Phi_{j}(t), j \in \mathbb{Z}$, be a sequence of functions of one variable $t$ satisfying

$$
\frac{d}{d t} \Phi_{j}(t)=\Phi_{j-1}(t), \quad j \in \mathbb{Z}
$$

Then we can construct a unique formal solution

$$
u(x)=\sum_{j=-\infty}^{\infty} u_{j}(x) \Phi_{j}(\varphi(x))
$$

of

$$
P(x, D) u(x)=0
$$

under the initial conditions

$$
\left.\frac{\partial^{k} u_{j}}{\partial x_{1}^{k}}\right|_{x_{1}=x_{1}{ }^{0}}=\delta_{j, 0} f_{k}\left(x^{\prime}\right), \quad k=0,1, \cdots, d-1, j \in \mathbb{Z},
$$

where $f_{k}\left(x^{\prime}\right)$ are arbitrary holomorphic functions defined on a neighborhood of $x^{0 \prime} . u_{j}(x)$ do not depend on the sequence $\Phi_{j}(t)$. Employing Hamada's method in [9], we can prove that $u_{j}(x)$ are holomorphic on a fixed complex neighborhood $V_{0}$ of $x^{0}$ and that there is a constant $C$ depending only on $f_{k}\left(x^{\prime}\right)$ such that

$$
\begin{gathered}
\left|u_{j}(x)\right| \leqq C^{j+1} j !, j \geqq 0 ; \\
\left|u_{j}(x)\right| \leqq \begin{cases}C^{-j+1}\left(\frac{\left|x_{1}-x_{1}{ }^{0}\right|^{-j}}{(-j) !}\right)^{\sigma /(\sigma-1)}, & \sigma>1, j<0, \\
0, & \sigma=1, j<0\end{cases}
\end{gathered}
$$

(see [17]). When $\sigma=1$, this is known since De Paris [5]; Hamada [9] obtains essentially the same estimates with the irregularity $\sigma$ replaced by the multiplicity $d$. 
The following proposition shows that (15) is the best estimates of this sort in general. It is not always, however, because $Q_{i}(x, \xi)$ in the De Paris decomposition may vanish on the subvariety $\{(x, \operatorname{grad} \varphi(x) \infty)\}$ of $N(K)$.

Proposition 1. If $\sigma>1$ and if $Q_{i}\left(x^{0}, \operatorname{grad} \varphi\left(x^{0}\right)\right)$ does not vanish for some $i<m$ with $d-d_{i}=\sigma(m-i)$, then there are data $f_{k}\left(x^{\prime}\right)$ such that we have

$$
\left|u_{j}(x)\right| \geqq c^{-j+1}\left(\left|x_{1}-x_{1}^{0 \mid}\right|^{-j} /(-j) !\right)^{\sigma /(\sigma-1)}
$$

on a neighborhood of $x^{0}$ with a constant $c>0$ for infinitely many $j<0$.

\section{§ 3. Hyperbolicily.}

From now on we assume that $P(x, D)$ is a linear differential operator with real analytic coefficients $a_{\alpha}(x)$ defined on an open set $\Omega$ in $\boldsymbol{R}^{n}$. Since the coefficients are continued analytically to a complex neighborhood $V$ of $\Omega$, all results in the preceding sections hold good. For the sake of simplicity we assume that $x^{0}=0=(0, \cdots, 0)$.

The operator $P(x, D)$ is said to be hyperbolic with respect to the hypersurface $S=\left\{x_{1}=0\right\}$ if the Cauchy problem

$$
\left\{\begin{array}{l}
P(x, D) u(x)=0 \\
\frac{\partial^{k-1} u\left(0, x^{\prime}\right)}{\partial x_{1}^{k-1}}=w_{k}\left(x^{\prime}\right), \quad k=1,2, \cdots, m,
\end{array}\right.
$$

is correctly posed. Of course, the hyperbolicity depends on the function space $\mathscr{F}$ to which the Cauchy data $w_{j}\left(x^{\prime}\right)$ and the solution $u(x)$ should belong. We consider three cases:

Case $I$. The space $\mathcal{E}$ of infinitely differentiable functions and dually the space $\mathscr{D}^{\prime}$ of distributions.

Case II. The spaces $\mathcal{E}^{(s)}$ and $\mathcal{E}^{\{s\}}$ of Gevrey classes of functions and the spaces $\mathscr{D}^{(s) \prime}$ and $\mathscr{D}^{\{s\}}$ of ultradistributions. Here $s>1$ and

$$
\begin{aligned}
& \mathcal{E}^{(s)}(\Omega)=\{f \in \mathcal{E}(\Omega) ; \forall K \subseteq \Omega \quad \forall / 2>0 \exists C(20)\}, \\
& \mathcal{E}^{\{s\}}(\Omega)=\{f \in \mathcal{E}(\Omega) ; \forall K \subseteq \Omega \quad \exists h \exists C(20)\},
\end{aligned}
$$

where 


$$
\sup _{x \in K}\left|D^{\alpha} f(x)\right| \leqq C h^{|\alpha|}(|\alpha| !)^{s}, \forall \alpha .
$$

$\mathscr{D}^{(s) \prime}(\Omega)$ and $\mathscr{D}^{\{s\}}(\Omega)$ are defined to be the duals of $\mathscr{D}^{(s)}(\Omega)=\mathscr{D}(\Omega)$ $\cap \mathcal{E}^{(s)}(\Omega)$ and $\mathscr{D}^{\{s\}}(\Omega)=\mathscr{D}(\Omega) \cap \mathcal{E}^{\{s\}}(\Omega)$ equipped with natural local convex topologies (see [16]).

Case III. The space $\mathcal{A}$ of real analytic functions and the space $\mathcal{B}$ of hyperfunctions.

If the coefficients of $P(x, D)$ are constant, a complete characterization of hyperbolic operators is known in each case (I. G. Petrowsky [32] and L. Gårding [7] for Case I; E. Larsson [19] and C. C. Chou [3] for Case II; T. Kawai [14] and P. Schapira [34] for Case III).

Therefore we will be concerned with the variable coefficient case. Usually the hyperbolicity condition consists of three parts:

Condition $A$. The initial surface is non-characteristic;

Condition $B$. The characteristic roots are real, i.e. the roots $\zeta_{1}$ of the algebraic equation

$$
p\left(x, \zeta_{1}, \xi^{\prime}\right)=0
$$

are real for any $x$ and $\xi^{\prime} \in \mathbb{R}^{n-1}$;

Condition $C$. Some conditions for lower order terms.

The necessity of Condition $\mathrm{A}$ is proved by constructing null-solutions. J. Hadamard [8] and S. Mizohata [25] proved the existence of $C^{k}$ nullsolutions for simple characteristic surfaces and De Paris [4] extended to the case of characteristic surfaces of irregularity 1 . We have proved the following theorem in [17] (cf. J. Persson [31]).

Theorem 1. Let $S: \psi(x)=0$ be a real analytic hypersurface such that $(x, \operatorname{grad} \psi(x) \infty)$ is a non-singular characteristic element for every $x \in S$. If $\sigma$ is the irregularity, then for each $1<s \leqq \sigma /(\sigma-1)$ and $x_{0} \in S$ there are null-solutions exactly in $\mathcal{E}^{\{s\}}$ and exactly in $\mathscr{D}^{(s) \prime}$ on a neighorhood of $x_{0}$.

Sketch of Proof. There is a holomorphic characteristic function $\varphi(x)$ with the same zeros $S$ as $\psi(x)$. Let $u_{j}(x)$ be the coefficients of formal solution (11) with the initial data $f_{k}\left(x^{\prime}\right)=\delta_{0, k}$. If we choose the sequence 


$$
\Phi_{j}(z)= \begin{cases}\int_{-i 0}^{z} \frac{(z-w)^{j-1}}{(j-1) !} \Phi_{0}(w) d w, & j>0, \\ \left(\frac{d}{d z}\right)^{-j} \Phi_{0}(z), & j \leqq 0,\end{cases}
$$

where

$$
\Phi_{0}(z)=\frac{-1}{2 \pi i} \int_{0}^{\infty} \frac{1}{z-t} d \exp \left(-t^{-1 /(s-1)}\right)
$$

or

$$
\Phi_{0}(z)=\exp (-i z)^{-1 /(s-1)},
$$

then it is shown that the formal solution (11) converges in the complex domain in the respective topology. The estimates (15) play an essential rôle. Lastly we take the boundary value of the solution on the real domain.

The necessity of Condition B in Case I was proved first by P. D. Lax [21] when the characteristic element is simple and then by S. Mizohata [24] without any restrictions on multiplicity. When the characteristic element is non-singular, we have

Theorem 2. Let $\left(0,\left(\zeta_{1}, \xi^{\prime}\right) \infty\right)$ be a non-singular characteristic element of irregularity 1 with $\xi^{\prime}$ real non-zero and $\operatorname{Im} \zeta_{1}>0$. Then for each $1<s<\infty$ there is a solution $u(x)$ of (17) exactly in $\mathcal{E}^{\{s\}}$ defined on the positive part $\Omega_{0}^{+}=\left\{x \in \Omega_{0} ; x_{1} \geqq 0\right\}$ of a neighborhood $\Omega_{0}$ of 0 with Cauchy data $w_{k}\left(x^{\prime}\right)$ having singularity spectra at $\left(0^{\prime}\right.$, $\left.\xi^{\prime} \infty\right)$ and such that $u(x)$ cannot be extended to any hyperfunction solution of $P(x, D) u(x)=0$ on any neighborhood of 0 .

Sketch of Proof. We construct a characteristic function $\varphi(x)$ with $\varphi(0)=0$ and $(0, \operatorname{grad} \varphi(0) \infty)=\left(0,\left(\zeta_{1}, \xi^{\prime}\right) \infty\right)$ and such that $\operatorname{Im} \varphi\left(0, x^{\prime}\right)$ $>0$ unless $x^{\prime}=0$. If we employ

$$
\Phi_{0}(z)=\frac{-1}{2 \pi i} \int_{0}^{\infty} \frac{1}{i z-t} d \exp \left(-t^{-1 /(s-1)}\right),
$$

and define $\Phi_{j}(z)$ by (22), then the formal solution converges as in the proof of Theorem 1 and turns out to be a solution exactly in $\mathcal{E}^{\{s\}}\left(\Omega_{0}^{+}\right)$ 
with the Cauchy data having singularity spectra at $\left(0^{\prime}, \xi^{\prime} \infty\right)$. It is actually real analytic on $\Omega_{0}^{+} \backslash\{0\}$.

If it were continued to a hyperfunction solution on a neighborhood of 0 , then it would follow from Holmgren's theorem and Sato's fundamental theorem [33] that the integral

$$
\int_{K^{\prime}} u\left(x_{1}, x^{\prime}\right) f\left(x_{1}, x^{\prime}\right) d x^{\prime}
$$

be continued real analytically to a fixed interval $(-\varepsilon, \varepsilon)$ for any real analytic function $f\left(x_{1}, x^{\prime}\right)$ on a neighborhood of $(-\varepsilon, \varepsilon) \times K^{\prime}$. But for a suitable $f\left(x_{1}, x^{\prime}\right)$ we can prove the divergence at $-\varepsilon / 2$.

Suppose that $\left(x,\left(\zeta_{1}, \xi^{\prime}\right) \infty\right)$ is a characteristic element with $\operatorname{Im} \zeta_{1}>0$ and $\xi^{\prime} \in \mathbb{R}^{n-1} \backslash\{0\}$. Since the singular part of $N(K)$ forms a subvariety of $N(K)$, we can find in an arbitrary small neighborhood of $\left(x,\left(\zeta_{1}, \xi^{\prime}\right) \infty\right)$ a non-singular characteristic element $\left(x^{0},\left(\zeta_{1}^{0}, \xi^{0 \prime}\right) \infty\right)$. Since the non-singular part of $N(K)$ is locally parametrized by $\left(x, \xi^{\prime} \infty\right)$, we may assume that $\xi^{\prime}$ is real and that $\operatorname{Im} \zeta_{1}^{0}>0$.

Now let $\left(0,\left(\zeta_{1}^{0}, \xi^{0 \prime}\right) \infty\right)$ be a non-singular characteristic element such that $\xi^{0 \prime}$ is real and $\operatorname{Im} \zeta_{1}{ }^{0}>0$. As in the proof of Theorem 2 we construct a holomorphic characteristic function $\varphi(x)$ satisfying $\varphi(0)=0$ and $(0, \operatorname{grad} \varphi(0) \infty)=\left(0,\left(\zeta_{1}{ }^{0}, \xi^{\prime \prime}\right) \infty\right)$. If $\varepsilon>0$ is sufficiently small, the domain $V_{0}$ of definition of the coefficients $u_{j}(x)$ of formal solution (11) around $x^{0}=(-\varepsilon, 0, \cdots, 0)$ contains 0 . Let

$$
\Phi_{0}(z)=\frac{-1}{2 \pi i} \frac{1}{z}
$$

and define $\Phi_{j}(z)$ similarly to (22). Then the solution $u(x)$ defined by (11) converges on the complex domain $\left\{x \in V_{0} ; \operatorname{Im} \varphi(x)>0\right\}$, which includes the real domain $\Omega_{0}{ }^{+}=\left\{x \in V_{0} \cap \boldsymbol{R}^{n} ; x_{1}>0\right\}$. On the other hand, we can prove in the same way as above that $u(x)$ can not be extended to any hyperfunction solution across the hyperplane $x_{1}=-\varepsilon$. If we shift the $x_{1}$ coordinate by $-\varepsilon$, we obtain the following

Theorem 3. Suppose that $P(x, D)$ has a non-singular characteristic element $\left(0,\left(\zeta_{1}, \xi^{\prime}\right) \infty\right)$ such that $\operatorname{Im} \zeta_{1}>0$ and $\xi^{\prime} \in \boldsymbol{R}^{n-1} \backslash\{0\}$. Then for any sufficiently small neighborhood $\Omega_{0}$ of 0 and any $\varepsilon>0$ 
there is a real analytic solution $u(x)$ of (12) defined on $\left\{x \in \Omega_{0} ; x_{1}\right.$ $>-\varepsilon\}$ which cannot be extended to any hyperfunction solution across the hypersurface $x_{1}=-2 \varepsilon$.

When the characteristic element is simple, H. Nakamura [28] has proved that there is a real analytic solution which cannot be extended to any ultradistribution solution on a larger domain.

We can generalize Theorem 2.2 of P. D. Lax [21] to the effect that if the Cauchy problem (17) has, for every $w_{k}\left(x^{\prime}\right) \in \mathcal{E}^{(s)}\left(\Omega^{\prime}\right)$ with a fixed domain $\Omega^{\prime}$ containing $0^{\prime}$, a solution $u(x) \in \mathscr{D}^{\{t\}^{\prime}}\left(\Omega_{w}\right)$ in some domain containing 0 , then there is a common domain $\Omega_{0}$ of existence for all solutions. Thus we obtain

Corollary. Let $\Omega^{\prime}$ be a domain containing $0^{\prime} \in \boldsymbol{R}^{n-1}$ and let $s$, $t>1$. If the Cauchy problem (17) has, for every $w_{k}\left(x^{\prime}\right) \in \mathcal{E}^{(s)}\left(\Omega^{\prime}\right)$ a solution $u(x) \in \mathscr{D}^{\{t\}}$ ' in some domain containing 0 , then the roots $\zeta_{1}$ of the equation

$$
p\left(0, \zeta_{1}, \xi^{\prime}\right)=0
$$

are real for any $\xi^{\prime} \in \boldsymbol{R}^{n-1}$.

K. Kataoka [13] has proved the necessity of Condition B for Case III by showing that if there is a characteristic root $\zeta_{1}\left(\xi^{\prime}\right)$ with $\operatorname{Im} \zeta_{1}<0$, then the Cauchy data $w_{k}\left(x^{\prime}\right)$ for solutions $u(x)$ in $x_{1}>0$ satisfy a pseudodifferential equation.

J. M. Bony-P. Schapira [1] proved that Conditions A and B are sufficient for hyperbolicity in Case III.

Originally Levi's condition $\sigma=1$ was introduced as Condition $\mathrm{C}$ in Case I by E. E. Levi [23], A. Lax [20], M. Yamaguti [35], S. MizohataY. Ohya [26] and J. Chazarain [2]. They proved that the Cauchy problem (17) is correctly posed in $\mathcal{E}$ and $\mathscr{D}^{\prime}$ if Conditions $\mathrm{A}$ and $\mathrm{B}$ hold and if every real characteristic element is non-singular and of irregularity 1 . Necessity of Levi's condition is also proved by S. MizohataY. Ohya [27] when the multiplicity $d$ is at most 2 and by H. Flaska-G. Strang [6] in the general case. More strongly we have the following 
Theorem 4. Suppose that Conditions $A$ and $B$ are satisfied and that $P(x, D)$ has a non-singular characteristic element $(0, \xi \infty)$ of irregularity $\sigma>1$.

Then for every $s>\sigma /(\sigma-1)$ there are Cauchy data $w_{k}\left(x^{\prime}\right) \in$ $\mathcal{E}^{(s)}\left(\Omega^{\prime}\right)$ on a neighborhood $\Omega^{\prime}$ of $0^{\prime}$ in $\mathbb{R}^{n-1}$ such that the solution $u(x)$ of (17) does belong to $\mathscr{D}^{(s) \prime}\left(\Omega_{0}\right)$ on any neighborhood $\Omega_{0}$ of 0 in $\mathbb{R}^{n}$.

On the other hand, for every neighborhood $\Omega$ of 0 in $\mathbb{R}^{n}$ there are Cauchy data $w_{k}\left(x^{\prime}\right) \in \mathcal{E}^{i s\}}\left(\Omega^{\prime}\right)$, where $s=\sigma /(\sigma-1)$ and $\Omega^{\prime} \supset \Omega \cap$ $\mathbb{R}^{n-1}$, such that the solution $u(x)$ of $(17)$ does not belong to $\mathscr{D}^{\{s\}}(\Omega)$.

Sketch of Proof. We construct a real valued real analytic characteristic function $\varphi(x)$ such that $\varphi(0)=0$ and $(0, \operatorname{grad} \varphi(0) \infty)=(0, \xi \infty)$. Since $N(K)$ is a real hypersurface, we rotate $\xi^{\prime}$ a little if necessary and may assume that the assumption of Proposition 1 is satisfied.

Suppose that for any Cauchy data $w_{k}\left(x^{\prime}\right) \in \mathcal{E}^{(s)}\left(\Omega^{\prime}\right)$ the solution $u(x)$ belongs to $\mathscr{D}^{(s) \prime}\left(\Omega_{w}\right)$ on some neighborhood $\Omega_{w}$ of 0 in $\mathbb{R}^{n}$. Then employing S. Mizohata's technique in his proof of Lemma 1.1 of [24], we can prove that there is a common neighborhood $\Omega_{0}$ of 0 such that all solutions $u(x)$ belong to $\mathscr{D}^{(s) \prime}\left(\Omega_{0}\right)$.

Thus we may assume that there are sufficiently small open sets $\Omega^{\prime} \subset \mathbb{R}^{n-1}$ and $\Omega_{0} \subset \mathbb{R}^{n}$ such that $0^{\prime} \in \Omega_{0} \cap \mathbb{R}^{n-1} \subset \Omega^{\prime}$ and that for any $w_{k}\left(x^{\prime}\right) \in \mathcal{E}^{*}\left(\Omega^{\prime}\right)$ the solution $u(x)$ of $(17)$ is in $\mathscr{D}^{* \prime}\left(\Omega_{0}\right)$, where $*$ is either $(s)$ or $\{s\}$, and will prove a contradiction.

Let $f \in \mathcal{E}^{*}(\mathbb{R})$. We restrict ourselves to the solutions $u(x)$ defined by (11), where we take

$$
\Phi_{j}(t)= \begin{cases}\int_{0}^{t} \frac{(t-s)^{j-1}}{(j-1) !} f(s) d s, & j>0 \\ \left(\frac{d}{d t}\right)^{-j} f(t), & j \leqq 0 .\end{cases}
$$

In view of (14) we can easily prove that

$$
u_{+}(x)=\sum_{j=0}^{\infty} u_{j}(x) \bar{\Phi}_{j}(\varphi(x))
$$

converges in $\mathcal{E}^{*}\left(\Omega_{0}\right)$. On the other hand, it follows from (15) that

$$
u_{-}(x)=\sum_{k=0}^{\infty} u_{-k}(x) \Phi_{-k}(\varphi(x))
$$


converges in $\mathscr{D}^{(\sigma /(\sigma-1)) \prime}\left(\Omega_{0}\right)$ and that the Cauchy data

$$
w_{k}\left(x^{\prime}\right)=\left.\frac{\partial^{k-1} u_{-}(x)}{\partial x_{1}^{k-1}}\right|_{x_{1}=0}, \quad k=1,2, \cdots, m
$$

belong to $\mathcal{E}^{*}\left(\Omega^{\prime}\right)$.

By the assumption $u_{-}(x)$ must belong to $\mathscr{D}^{* \prime}\left(\Omega_{0}\right)$ for every $f \in$ $\mathcal{E}^{*}(\boldsymbol{R})$. We consider the linear mapping $T: \mathscr{D}^{*}(\boldsymbol{R}) \rightarrow \mathscr{D}^{* \prime}\left(\Omega_{0}\right)$ defined by $T f=u_{-}$. Since $T$ is continuous as a mapping from $\mathscr{D}^{*}(\boldsymbol{R})$ into $\mathscr{D}^{(\sigma /(\sigma-1)) \prime}\left(\Omega_{0}\right)$, it has a closed graph and hence is continuous. As we will prove in [18], the kernel theorem holds for the ultradistributions of class $*$. Hence there is an ultradistribution $T(t, x) \in \mathscr{D}^{* \prime}\left(\boldsymbol{R} \times \Omega_{0}\right)$ such that

$$
u_{-}(x)=T f(x)=\int T(t, x) f(t) d t
$$

We may assume without loss of generality that $\varphi(x)=x_{n}$. Then we can write

$$
u_{-}(x)=T f(x)=\sum_{k=0}^{\infty} u_{-k}(x) \frac{d^{k}}{d x_{n}{ }^{k}} f\left(x_{n}\right) .
$$

Hence it follows that the support supp $T$ of the kernel is contained in the hyperplane $\left\{(t, x) \in \boldsymbol{R} \times \Omega_{0} ; t=x_{n}\right\}$. By the structure theorem of ultradistributions with support in a submanifold (see [18]) we have

$$
T(t, x)=\sum_{k=0}^{\infty} v_{k}(x) \delta^{(k)}\left(x_{n}-t\right),
$$

where $v_{k}(x)$ are ultradistributions in $\mathscr{D}^{* \prime}\left(\Omega_{0}\right)$ satisfying the following estimates:

In case $*=(s)$ (resp. $*=\{s\})$, we can find, for every compact set $K$ in $\Omega_{0}$, constants $h, L$ and $C$ (resp. for every compact set $K$ in $\Omega_{0}$ and $h, L>0$, a constant $C$ ) such that

$$
\left\|v_{k}(x)\right\|_{\left(\mathscr{D}_{K}^{\{s\}, h},\right)^{\prime}} \leqq C L^{k} /(k !)^{s} .
$$

Comparing (33) and (34), we have $v_{k}(x)=u_{-k}(x)$. Then the estimates (35) contradict (16).

If we wanted to prove only the necessity of Levi's condition, we needed only the Schwartz kernel theorem and the Schwartz structure theorem of distributions with support in a submanifold. Then the proof 
is very similar to J. Peetre's characterization of linear differential operators [30].

V. Ya. Ivrii-V. P. Petkov [12] and L. Hörmander [11] investigate necessary conditions for hyperbolicity in $\mathcal{E}$ at singular characteristic elements.

As we remarked above Levi's condition $\sigma=1$ is known to be sufficient for hyperbolicity in Case I when there are no singular characteristic elements. In case II we have

Theorem 5. The Cauchy problem (17) is correctly posed in $\mathcal{E}^{(s)}$ and $\mathscr{D}^{(s) \prime}$ (resp. in $\mathcal{E}^{\{s\}}$ and $\mathscr{D}^{\{s\}}$ ) if Conditions $A$ and $B$ hold and if every real characteristic element is non-singular and of irregularity $\sigma \leqq s /(s-1) \quad($ resp. $\sigma<s /(s-1))$.

A little weaker results have been obtained by Y. Ohya [29], J. Leray-Y. Ohya [22] and Y. Hamada-J. Leray-C. Wagschal [10]. Theorem 5 may be proved by each of their methods. We can also prove it by constructing the fundamental solution as the superposition of Hamada's solutions [9] with polar Cauchy data as in T. Kawai [15]. Again estimates (15) play an essential rôle.

\section{References}

[1] J.-M. Bony et P. Schapira: Solutions hyperfonctions du problème de Cauchy, Hyperfunctions and Pseudo-Differential Equations, Lecture Notes in Math., 287, Springer, Berlin-Heiddlberg-New York, 1973, pp. 82-98.

[2] J. Chazarain: Opérateurs hyperboliques à caractéristiques de multiplicité constante, Ann. Inst. Fourier, Grenoble, 24 (1974), 173-202.

[3] Ch.-Ch. Chou: La Transformation de Fourier Complexe et l'Équation de Convolutions, Lecture Notes in Math., 325, Springer, Berlin-Heidelberg-New York, 1973.

[4] J.-C. De Paris: Problème de Cauchy oscillatoire pour un opérateur différentiel à caractéristique multiples; lien avec l'hyperbolicités, J. Math. Pures Appl. 51 (1972), 231-256.

[5] J.-C. De Paris: Problème de Cauchy analytique à données singulières pour un opérateur différentiel bien décomposable, J. Math. Pures Appl. 51 (1972), 465-488.

[6] H. Flaska and G. Strang: The correctness of the Cauchy problem, Advances in Math. 6 (1971), 347-379.

[7] L. Gårding: Linear hyperbolic partial differential equations with constant coefficients, Acta Math. 85 (1950), 1-62.

[8] J. Hadamard: Le Problème de Cauchy et les Équations aux Dérivées Partielles Linéaires Hyperboliques, Hermann, Paris, 1932.

[9] Y. Hamada: Problème analytique de Cauchy à caractéristiques multiples dont les 
données de Cauchy ont des singularités polaires, C. R. Acad. Sci. Paris, Ser. A, 276 (1973), 1681-1684.

[10] Y. Hamada, J. Leray et C. Wagschal: Systèmes d'équations aux dérivées partielles à caractéristiques multiples: Problème de Cauchy ramifié; Hyperbolicité partielle, J. Math. Pures Appl. 55 (1976), 297-352.

[11] L. Hörmander: On the Cauchy problem for differential equations with double characteristics, to appear.

[12] V. Ya. Ivrii and V. P. Petkov: Necessary conditions for the correctness of the Cauchy problem for non-strictly hyperbolic equations, Usp. Mat. Nauk, 19 (170) (1974), 3-70 (in Russian).

[13] K. Kataoka: Radon transforms of hyperfunctions and their applications, Master's thesis, Univ. of Tokyo, 1976 (in Japanese).

[14] T. Kawai: On the theory of Fourier hyperfunctions and its applications to partial differential equations with constant coefficients, J. Fac. Sci. Univ. Tokyo, Sect. IA, 17 (1970), 467-517.

[15] T. Kawai: Construction of local elementary solutions for linear partial differential operators with real analytic coeffients (I) - The case with real principal symbolsPubl. RIMS, Kyoto Univ. 7 (1971), 363-397.

[16] H. Komatsu: Ultradistributions, I. Structure theorems and a characterization, J. Fac. Sci. Univ. Tokyo, Sect. IA, 20 (1973), 25-105.

[17] H. Komatsu: Irregularity of characterististic elements and construction of null-solutions, J. Fac. Sci. Univ. Tokyo, Sect. IA, 23 (1976), 297-342.

[18] H. Komatsu: Ultradistributions, II. The kernel theorem and ultradistribution with support in a submanifold, J. Fac. Sci. Univ. Tokyo, Sect. IA, to appear.

[19] E. Larsson: Generalized hyperbolicity, Ark. f. Mat. 7 (1967), 11-32.

[20] A. Lax: On Cauchy's problem for partial differential equations with multiple characteristics, Comm. Pure Appl. Math. 9 (1956), 135-169.

[21] P. D. Lax: Asymptotic solutions of oscillatory initial value problems, Duke Math. J. 24 (1957), 627-646.

[22] J. Leray et Y. Ohya: Systèmes linéaires, hyperboliques non stricts, Colloque CBRM, 1964, pp. 105-144.

[23] E. E. Levi: Caratteristiche multiple e problema di Cauchy, Ann. di Mat. 16 (1909), 161-201.

[24] S. Mizohata: Some remarks on the Cauchy problem, J. Math. Kyoto Univ. 1 (1961), 109-127.

[25] S. Mizohata: Solutions nulles et solutions non analytiques, J. Math. Kyoto Univ. 1 (1962), 271-302.

[26] S. Mizohata et Y. Ohya: Sur la condition de E. E. Levi concernant des équations hyperboliques, Publ. RIMS, Kyoto Univ., Ser. A, 4 (1968), 511-526.

[27] S. Mizohata et Y. Ohya: Sur la condition d'hyperbolicité pour les équations à caractéristiques multiples, II, Japan. J. Math. 40 (1971), 63-104.

[28] H. Nakamura: A necessary condition for hyperbolic equations, Master's thesis, Univ. of Tokyo, 1975 (in Japanese).

[29] Y. Ohya: Le problème de Cauchy pour les équations hyperboliques à caractéristique multiple, J. Math. Soc. Japan, 16 (1964), 268-286.

[30] J. Peetre: Une caractérisation abstraite des opérateurs différentielles, Math. Scand. 7 (1959), 211-218 and 8 (1960), 116-120.

[31] J. Persson: Non-uniqueness in the characteristic Cauchy problem when the coefficients are analytic, Matematiche, 27 (1972), 1-8.

[32] I. G. Petrowsky: Über das Cauchysche Problem fur ein System linearer partieller Differentialgleichungen in Gebiete der nichtanalytischen Funktionen, Bull. Univ. État. Moscou 1 fasc. 7 (1938), 1-74. 
[33] M. Sato, T. Kawai and M. Kashiwara: Microfunctions and psedo-differential equations, Hyperfunctions and Pseudo-Differential Equations, Lecture Notes in Math., 287, Springer, Berlin-Heidelberg-New York, 1973, pp. 265-529.

[34] P. Schapira: Théorème d'unicité de Holmgren et opérateurs hyperboliques dans l'espace des hyperfonctions, An. Acad. Brasil. Ci. 43 (1971), 39-44.

[35] M. Yamaguti : Le problème de Cauchy et les opérateurs d'intégrale singulière, Mem. Coll. Sci., Kyoto Univ. 32 (1959), 121-151. 
